arte contemporáneo, exponiendo a su vez la problemática que conlleva la conservación de este tipo de piezas. Virginia Costa, representando al Laboratorio de Investigación de los Monumentos Históricos franceses (LRMH), repasó las técnicas electroquímicas aplicadas a la conservación de metales y como modificar el método para un uso más adecuado.

En representación de Rocco Mazzeo (Universidad de Bolonia), Paola Letardi, del Instituto de Ciencias Marinas de Génova, propuso una mayor investigación de los problemas de corrosión de bronces expuestos al exterior. La sección de Metales de la Escuela Superior de Amberes, dirigida por Patrick Storme, mantiene una politica de investigación que mostró en el seminario como modelo de programa de formación en conservaciónrestauración de metal. El Dr. Christian Degrigny, coordinador del grupo de trabajo Metal del ICOMComité para la Conservación, comentó el dinamismo y el esfuerzo que el Consejo Internacional de Museos hace para conseguir más dialogo. En el espacio reservado para A-RSF se presentó la primera parte de las actividades de la asociación en Costa Rica, el Proyecto Alajuela, que se llevó a cabo en el verano 2003. En dicho proyecto participaron Montse Artigau, profesora de restauración de materiales inorgánicos en la Escuela de Conservación y Restauración de BB CC de Barcelona, y Margarida Silva, restauradora-conservadora, quienes presentaron el trabajo realizado: restauración e investigación de una fuente de metal del siglo XIX.

Finalmente, la mesa redonda, moderada por Regis Bertholon y Juan Carlos Bermejo, se dirigió hacia dos vertientes: el patrimonio industrial y la corrosión de metales expuestos al aire libre, debate que contó con la presencia de Herman
Terryn de la VUB (Universidad Libre de Bruselas), quien habló de casos tan conocidos como el estudio del Atomium; de Immaculada Aguilar, que habló del Programa de Conservación del Patrimonio Industrial Español y de la necesidad de la catalogación para la conservación de todos los elementos del patrimonio industrial, y de Myriam Serck-Dewaide, quien propuso una colaboración entre los especialistas de patrimonio industrial y el IRPA, para poder avanzar en la investigación.

El tercero y último día, se hizo una visita al Museo de Arte Antiguo de Namur para conocer la exposición "Alrededor de Hugo d 'Oignies", artista que realizó las piezas de orfebrería mosana pertenecientes al Tesoro del Antiguo Priorato de San Nicolás, fundado a principios del siglo XIII en Oignies, cerca de Charleroi, Bélgica.

El encuentro se caracterizó por la buena atmósfera, la calidad de las comunicaciones y la participación constante del público. Como muestra de los buenos resultados, se anunció el III Seminario Internacional A-RSF, que se celebrará en Valencia en el 2005, esta vez bajo el tema de Conservación de Interiores Históricos.

Nos gustaría agradecer a Regis Bertholon su ayuda durante todo el seminario y a Myriam Serck-Dewaide por poner a disposición de la Asociación Restauradores sin Fronteras las instalaciones del IRPA.

Juan Carlos Bermejo Cejudo y Raquel Ballester García

Coordinadores del II Seminario

Internacional A-RSF

\title{
El Algarve y Andalucia dialogan sobre patrimonio y museología
}

Las VII Jornadas de Patrimonio del Algarve, organizadas inicialmente por la Cámara Municipal de Albufeira y, desde hace dos años, en colaboración con la Universidad de Algarve, se celebraron los días 23 y 24 de octubre de 2003 en Albufeira.

Hacer un balance sobre el desarrollo de las VII Jornadas pasa por destacar en primer término el diálogo que se estableció entre el Algarve y Andalucia, dos regiones limítrofes que a pesar de su cercanía no encuentran frecuentemente escenarios donde exponer experiencias paralelas. Así, podríamos extraer como puntos de conclusión, que serán detallados en la publicación de las Actas de las Jornadas que se está gestionando, la buena iniciativa de hacer extensivo el tema de las jornadas -la Museología- a nuestro país, acor- tar distancias (que realmente en cuanto al modo de trabajar no son tales) y destacar el interés por el acercamiento a nuestra realidad patrimonial, que también deberíamos no sólo valorar sino fomentar desde nuestras posibilidades por la riqueza que pueda aportarnos a todos.

Hay que valorar este interés de la comunidad portuguesa por aproximarse a España, a Andalucía, por intercambiar y poder confluir en proyectos comunes, especialmente en el ámbito de la educación en los museos.

$M^{\mathrm{a}}$ Teresa Suárez Domínguez Historiadora del Arte, especializada en Museos y Patrimonio 